\title{
Treatment of steroid-resistant nephrotic syndrome
}

\begin{abstract}
Nephrotic syndrome is the most common glomerular disorder in children, and corticosteroids are the first choice of treatment. While the majority of children respond to corticosteroid therapy, a few do not enter remission after daily therapy for 1-2 months, hence showing steroid-resistance. Most of these children show focal and segmental glomerulosclerosis (FSGS) upon renal biopsy. Steroid resistance and associated complications make management of these patients very challenging, with their higher chance of progression to end-stage renal failure. Various immunosuppressive drugs have been used to induce remission with different success rates, though most of them have shown weak effectiveness. However, careful weighing of their toxic effects and effective dose should be done, especially when treating children with a non-malignant disease like nephrotic syndrome, thus obtaining optimum results. This review discusses steroid-resistant nephrotic syndrome and treatment strategies that have been attempted.
\end{abstract}

Volume 5 Issue I - 2017

\author{
Asiri S Abeyagunawardena,' UI Karunadasa,' \\ S Abeyagunawardena ${ }^{2}$ \\ 'Department of Pediatrics, University of Peradeniya, Sri Lanka \\ ${ }^{2}$ Out-patient Department of Medicine, Teaching Hospital \\ Peradeniya, Sri Lanka
}

\begin{abstract}
Correspondence: AS Abeyagunawardena, Professor of Pediatrics, Department of Pediatrics, Faculty of Medicine, University of Peradeniya, Sri Lanka, Tel 94777843848 , Email asiriabey26@gmail.com
\end{abstract}

Received: January 06, 2017 | Published: June 26, 2017

Keywords: steroid-resistant nephrotic syndrome, corticosteroid therapy, immunosuppressive treatment, toxicity, membranoproliferative glomerulonephritis

Abbreviations: NS, nephrotic syndrome; FSGS, focal and segmental glomerulosclerosis; MCD, Minimal change disease; ESR, erythrocyte sedimentation rate; HIV, human immunodeficiency virus; CYC, cyclophosphamide; CHL, chlorambucil; MMF, mycophenolate mofetil; MPGN, membranoproliferative glomerulonephritis; SDNS, steroid-dependent nephrotic syndrome; FRNS, frequently-relapsing nephrotic syndrome; SRNS, steroid-resistant nephrotic syndrome

\section{Introduction}

Nephrotic syndrome (NS) is characterized by heavy proteinuria, hypoalbuminemia, edema and hyperlipidemia. The syndrome can be sub-classified as congenital, primary and secondary forms. ${ }^{1}$ The majority of children with primary disease have minimal changes in the glomeruli on histology, and $90-95 \%$ will respond corticosteroid therapy. ${ }^{2}$ In children unlike in adults, it is not routine practice to perform renal biopsy at the initial presentation of NS, unless atypical features such as macroscopic haematuria, renal impairment or persistent severe hypertension are present. Most pediatric nephrologists will consider a biopsy in children who fail to respond to glucocorticoid therapy within 1-2 months. The great majority of these biopsies show either focal and segmental glomerulosclerosis (FSGS) or minimal change disease (MCD). These are probably not distinct clinical and pathological entities but represent part of a disease spectrum ranging from highly steroid-sensitive patients with MCD at one end and children with rapidly progressive FSGS, unresponsive to any form of treatment, at the other. It should be emphasized that FSGS is a histological pattern that can occur as the result of several diseases and syndromes other than the NS (e.g. advanced reflux nephropathy, cyclosporine A toxicity etc), and not a disease entity by itself. ${ }^{3}$

There is some evidence that children showing lesions of FSGS early in the course of the disease are less responsive both to steroids and to other drugs than those with MCD. ${ }^{4,5}$ Moreover, some patients with MCD on the first biopsy may have FSGS if a biopsy is done later in the disease, especially if they are persistently steroid resistant or if they have developed secondary steroid resistance. ${ }^{6}$ Since it is only necessary to identify a single area of focal hyalinosis in a single glomerulus to diagnose FSGS, the question of whether FSGS was present all along but missed on the first biopsy because a representative lesion was not included in the biopsy, or whether MCD can evolve into FSGS, is not clear. Primary idiopathic FSGS has no demonstrable etiological factor and there are no universally accepted clinical criteria or histological features that predict a poor response to immunosuppressive therapy. The presence of tubulointerstitial lesions and wide spread capillary loop collapse on renal histology are generally thought to be associated with poor response to immunosuppressive therapy, whereas the presence of mesangial hypercellularity or tip lesions may indicate a more favorable response. However the main reason for performing a biopsy after initial failure to respond to steroids is to exclude one of the rarer causes of NS, such as Membranoproliferative glomerulonephritis or rapidly progressive glomerulonephritis, for which a different treatment strategy would be appropriate.

Steroid-resistant NS is a therapeutic challenge for the Pediatrician or the Pediatric Nephrologists', and there is currently a debate regarding the optimal therapy for such patients. Although treatment for this group of patients is far from satisfactory, an important guiding principle stems from the fact that children who have refractory proteinuria have a very poor prognosis, with a higher propensity for progression to end stage renal failure, perhaps $50 \%$ within $5-10$ years. ${ }^{7}$ It is therefore justified to try to induce a remission by the empirical use of immunosuppressive drugs of various types, even when (as is usually the case) the evidence for drug effectiveness is weak. In spite of toxic side effects, if these drugs are used carefully, the side-effects can be minimized, and the gain to the patient if treatment is successful is phenomenal. On the other hand one should exercise caution when prescribing immunosuppressive therapy as failure to achieve remission results only in renal death and over immunosuppression can lead to the death of the child.

If a child fails to enter complete remission following the administration of daily prednisolone $60 \mathrm{mg} / \mathrm{m} 2 /$ day for four weeks then a renal biopsy should be performed. ${ }^{8}$ Moreover, following investigations are generally performed to screen for secondary causes of NS.
a. Anti streptolysin O titer
b. ESR 


\section{c. C3 and $\mathrm{C} 4$}

d. Anti nuclear antibody and double stranded DNA

e. Screening for Hepatitis B

f. HIV screening (optional)

If the investigations exclude a secondary cause for NS then depending upon the renal histology other immunosuppressive therapy will be considered.

\section{Available drug therapy \\ Prolonged steroid treatment}

The initial response to conventional doses of glucocorticoid therapy for idiopathic FSGS is poor in contrast to that of minimal change glomerulopathy. ${ }^{9}$ In a majority of studies published, the response rate has been less than $30 \% .{ }^{10}$ Pei et al. ${ }^{11}$ reported that, using a more prolonged course of prednisolone therapy, $44 \%$ of children with idiopathic FSGS entered complete remission. The available evidence on the efficacy of such treatment is inconsistent and therefore it is more appropriate, effective and more gentle to the patient to introduce other immunosuppressive drugs at this juncture.

\section{High-dose intravenous methylprednisolone}

Methylprednisolone administered intravenously either daily or on alternate days at a dosage of $1 \mathrm{~g} / 1.73 \mathrm{~m} 2$ body surface areas to a total of 3-6 doses is effective in the treatment of renal allograft rejection and some forms of rapidly progressive glomerulonephritis. ${ }^{12}$ It has succeeded in a small number of cases in inducing remission in children with NS who have not responded to a conventional course of oral steroids..$^{13-15}$ This treatment is usually well tolerated, especially if given on alternate days, and some prefer to try this approach before exposing patients to the multiple toxic side-effects of the other drugs discussed below. However, many clinicians will be concerned about the long-term exposure to steroids which is already so great and would reserve intravenous methylprednisolone for combination therapy with an alkylating agent at a later stage.

\section{Alkylating agents}

Reliable data on the use of alkylating agents in SRNS are scanty, making evidence-based recommendations difficult to formulate. Evidence suggests that steroid-resistant children with MCD are more likely to respond than those with FSGS. ${ }^{5}$ Because of the potentially serious consequences of failure to induce remission of proteinuria, it is worth a trial of a standard course of cyclophosphamide (CYC) before abandoning a child to the prospect of renal replacement therapy. Additional strength is given to this argument because children with FSGS who receive transplants have a high incidence of early recurrence of their original disease in the graft, often leading to graft loss. One study group has treated children with SRNS with a prolonged course of intravenous methylprednisolone, combined with chlorambucil (CHL) or CYC if the steroid alone failed to induce a remission after 10 weeks of treatment.

Methylprednisolone was given as six alternate-day doses of $30 \mathrm{mg} /$ $\mathrm{kg}$ during the first 2 weeks, then weekly in the same dose for a further 8 weeks, then fortnightly for a further 8 weeks, then monthly for a further 32 weeks and finally alternate months for a further 25 weeks ( 78 weeks in all). After the first 2 weeks of treatment, the children were also given oral prednisone, $2 \mathrm{mg} / \mathrm{kg}$ on alternate days until the end of the course. The alkylating agents were given as either CYC
$2 \mathrm{mg} / \mathrm{kg} /$ day or CHL $0.2 \mathrm{mg} / \mathrm{kg} /$ day for $8-12$ weeks. Of 23 children described in the original report, 12 went into complete, sustained remission, six lost their NS but remained proteinuric, four remained nephrotic, and one died in chronic renal failure. Fifteen of the 23 had at least one course of an alkylating agent. All patients had FSGS either in the original biopsy or in later biopsies. Although these results are impressive, the regimen is potentially very toxic and is perhaps best reserved for selected cases. ${ }^{16}$

\section{Cyclosporin A}

Cyclosporin A has successfully induced remission in children with SRNS due to FSGS, in whom previous attempts to control the disease with alkylating agents had failed. Increasingly CsA has been used as second-line treatment for corticosteroid-resistant FSGS, with supportive evidence from randomized controlled trials. ${ }^{17} \mathrm{~A}$ higher proportion of sustained remissions have been achieved with CsA administered in conjunction with alternate day corticosteroids than CsA alone [18-20]. CsA was given in a starting dose of $150 \mathrm{mg} / \mathrm{m} 2 /$ day or 3-5 $\mathrm{mg} / \mathrm{kg}$ in two divided doses, adjusted to achieve a trough plasma CsA concentration of 100-200 ng/ml. Prednisone is given at $30 \mathrm{mg} / \mathrm{m} 2 /$ day, also in two divided doses, for the first month, followed by $30 \mathrm{mg} / \mathrm{m} 2$ on alternate days for 3-6 months. ${ }^{21}$ However, many children relapse following discontinuation of CsA therapy, making them CsA dependant or increasing steroid sensitivity in steroidresistant children. ${ }^{22}$ In conclusion, it remains unclear whether CsA therapy improves long-term renal survival despite the encouraging short-term success in induction of remission in SRNS due to FSGS.

\section{Levamisole}

There is no evidence that levamisole has any beneficial effect in SRNS. ${ }^{23}$

\section{Tacrolimus}

Complete or partial remission in patients who were resistant to both steroids and cyclosporine when treated with tacrolimus has been reported infrequently in the literature. However most studies included a small number of patients ${ }^{24,25}$ Controlled data are not available and this drug should be considered experimental at present.

\section{Vincristine}

A handful of children with steroid and alkylating agent-resistant nephrotic syndrome have lost their proteinuria following treatment with the antibiotic alkaloid vincristine. ${ }^{26,27}$ In most cases the drug was given with steroids and a second or third course of an alkylating agent. It is therefore impossible to be certain whether the success of the treatment was due to vincristine or the other simultaneously administered drugs. Vincristine is neurotoxic and must be given intravenously, its effect on tissues being similar to that of mustine if extravasation occurs. ${ }^{28}$ It cannot be said to have an established place in the management of SRNS but encouraging results are emerging.

\section{Rituximab}

The chimeric anti-CD20 monoclonal antibody Rituximab is used for B cell lymphomas. The mechanism of action of rituximab in NS is not yet clearly illustrated; however one hypothesis says that its effect on $\mathrm{T}$ cells causes a lasting effect to reduce proteinuria. ${ }^{29-30}$ Several studies reported their results for rituximab use in steroid-dependent (SDNS), frequently-relapsing (FRNS) and steroid-resistant NS. From these, results for SDNS are the most successful with 12-16 months sustained remission whereas they are less effective but still 
encouraging in SRNS patients..$^{31-35}$ Bagga et al..$^{36}$ reported complete remission in 3 out of 5 patients treated with rituximab, with significant increase of serum album and no side effects. In contrast, Magnasco et al. ${ }^{37}$ state that rituximab gives no benefit when used as add-on therapy to steroids and calcenurin inhibitors in SRNS. Though rituximab seems to be a potent drug in NS, further randomised studies would definitely shed more light on its use in SRNS.

\section{Mycophenolatemofetil}

Mycophenolate mofetil (MMF) can inhibit the de novo pathway of guanosine nucleotide synthesis which is important in the proliferation of T and B-lymphocytes. Since MMF is neither nephrotoxic nor gonadotoxic it might seem a good option to treat children who show these adverse effects due to prolonged treatment. ${ }^{38}$ Though no many studies have been done to investigate MMF's efficacy in SRNS, Briggs et al in 1998 reported reduction of proteinuria by MMF better than cyclosporine A in a small group of SRNS patients with major steroid-sparing effects and no toxicity. ${ }^{39} \mathrm{~A}$ more recent study by $\mathrm{Li}$ et al. ${ }^{40}$ suggests that MMF could be more effective in children with SRNS under the age of 2 years. ${ }^{40}$

\section{Toxicity of cytotoxic and immunosuppressive drugs: a pediatric perspective}

The toxicity of cytotoxic drugs such as CYC and CHL has been well documented for many years, and include short-term as well as long-term toxicity. Bone marrow suppression with leucopenia, anemia, and thrombocytopenia, alopecia, nausea, abdominal pain, and hemorrhagic cystitis (for CYC), constitute the most important short-term side effects. Arterial hypertension, hypertrichosis, gingival hyperplasia, hypomagnesaemia, hyperuricemia, and nephrotoxicity may be encountered during CYA therapy. ${ }^{41-44}$ Infection is a universal concern in patients receiving cytotoxic or immunosuppressive therapy. Concomitant glucocorticoid therapy adds to this problem and should be administered as an alternate day regimen wherever possible. A detailed account of the plethora of opportunistic infections that can occur is beyond the scope of this article, however risk of infection with cytomegalovirus, Pneumocystis carinii, and varicella zoster $^{45}$ remains ever present. ${ }^{46,47}$

Of particular concern regarding the use of cytotoxic drugs is the long-term cancer risk although this risk has not been quantified in children. ${ }^{48,49}$ There is generally a lack of data regarding cumulative dose toxicity for the various agents mentioned above, but this is an important factor to bear in mind and should be discussed with parents and child before the onset of therapy. Other potential medium and long-term side effects such as teratogenicity and infertility are also important considerations although in reality rarely become a major concern at the doses and durations of cytotoxic immunosuppression employed in the treatment of childhood NS. ${ }^{50,51}$ Cytotoxic and immunosuppressive drugs undoubtedly play an important role in the treatment of certain forms of childhood NS, although these drugs in themselves are associated with significant morbidity and even mortality. It is therefore of particular importance that the benefits and risks of these agents are weighed when considering their use in the treatment of non-malignant diseases.

\section{Membranoproliferative glomerulonephritis (MPGN)}

(mesangiocapillary)

This disease is uncommon in children and very rare in the first decade. It typically presents with haematuria (macroscopic or microscopic), non-selective proteinuria which is usually, but not always in the nephrotic range, and frequently hypertension and deteriorating renal function. Hypocomplementemia (persistently reduced plasma concentration of $\mathrm{C} 3$ ) is commonly seen associated with $\mathrm{MPG}^{52}$ Pedia the traditional classification of MPGN divided the disease into three subtypes as MPGN I, II and III depending histological features. The newer pathogenesis-based classification of MPGN depends on immunofluorescence (IF) staining, and hence identifies MPGN types as follows;

\section{i. Immune complex-mediated MPGN}

ii. Complement-mediated MPGN/C3 glomerulopathy (C3 dominant IF)

iii. MPGN not related to complement or immune complex

\section{iv. Negative IF}

Depositions of immunocomplexes can be observed in Immune complex-mediated MPGN. Frequent infections that result in this type are Hepatitis C and B. Systemic lupus erythematosus, sclerodermia, Sjögren syndrome and rheumatoid arthritis are the most common autoimmune diseases that cause MPGN due to persistently circulating immunocomplexes. ${ }^{53,54} \mathrm{C} 3$ glomerulonephritis $(\mathrm{C} 3 \mathrm{GN})$ and dense deposit disease (DDD) are the two subtypes of $\mathrm{C} 3$ glomerulopathy. Prominent deposition of $\mathrm{C} 3$ in the mesangium and capillary wall is seen in C3GN whereas electron-dense deposits in the lamina densa of the glomerular basement membrane are the defining feature of DDD, which was earlier known as MPGN type $2 .{ }^{55}$ Angiotensin-converting enzyme (ACE) inhibitors and angiotensin II receptor blockers are prescribed to reduce proteinuria, and non-specific strategies such as controlling the blood pressure and lowering serum lipid levels would also have beneficial effects in patients having $\mathrm{C} 3$ glomerulopathy. ${ }^{56}$ Eculizumab, a monoclonal antibody which inhibits the cleaving of C5 has been used as a targeted therapy to block the complement cascade. However, its effect has been reported only in reports on single patients and one study that included 6 patients. ${ }^{57}$

A controlled study of 5 years alternate-day prednisone by the ISKDC showed little or no overall advantage of treatment over control, although a small benefit was claimed for patients of types 1 and $3 .^{58}$ Data on the efficacy of cyclophosphamide in MPGN are quite conflicting since a study by Cattaran et al. ${ }^{59,60}$ showed no difference in survival and renal function compared to the control group whereas an uncontrolled study by Faedda et al. ${ }^{61,62,63}$ showed $79 \%$ remission rate after 10 months treatment with cyclophosphamide and prednisolone. Data are limited for the efficacy of cyclosporine A, tacrolimus and mycophenolate mofetil in immunoglobulinassociated MPGN. Excellent results were claimed in patients with type 1 membranoproliferative disease for a regimen beginning with six alternate-day high-dose infusions of methylprednisolone followed by alternate-day oral prednisone for 1-5 years. The aim of methylprednisolone infusions is to promote early stabilization of the disease. Due to the reclassification and emergence of new knowledge on the pathophysiology of MPGN, it seems advisable not to consider very early studies on treatment for this disease, since the study cohorts could have been a mixture of patients with different path biologic conditions. More targeted therapies based on current knowledge should be considered after evaluation.

\section{Conclusion}

Steroid-resistant nephrotic syndrome currently does not have a precise treatment guideline for optimal therapy, and is therapeutically challenging. Though aggressive steroid therapy 
seems to resolve proteinuria in some patients, it would be wiser to use immunosuppressive drugs at this point. Renal histology should also be taken into account when deciding treatment strategies for SRNS patients. Cyclosporine has shown successful short-term remission rates with SRNS children, whereas alkylating agents and other immunosuppressive therapy used in steroid-sensitive disease have not shown much efficacy. This could be due to the scarce amount of studies done on their action in SRNS, with less numbers of patients. However, patients should be monitored for toxic side effects of immunosuppressive therapy, especially infections. More future research on this area would definitely aid in formulating proper guidelines for the treatment of SRNS.

\section{Acknowledgments}

None.

\section{Conflicts of Interset}

None.

\section{References}

1. Niaudet P. Steroid-sensitive nephrotic syndrome in children. In: ED Avner, et al., editors. Pediatric Nephrology. USA: Lippincott Williams and Wilkins, USA, 2004. p. 43-556.

2. Trachtman H, Sampson M, Sethna C. Childhood onset Nephrotic syndrome. In: Fervenza F, et al., editors. Core Concepts in Parenchymal Kidney Disease. Springer Science \& Business Media, USA, 2013. p. 23-24.

3. Sprangers B, Meijers B, Appel G. FSGS: Diagnosis and Diagnostic Work-Up. Biomed Res Int. 2016;2016:4632768.

4. Fogo A. Causes and pathogenesis of focal segmental glomerulosclerosis. Nat Rev Nephrol. 2014;11:76-87.

5. A Report of the International Study of Kidney Disease in Children. The primary nephrotic syndrome in children. Identification of patients with minimal change nephrotic syndrome from initial response to prednisone. $J$ Pediatr. 1981;98(4):561-564.

6. Banaszak B, Banaszak P. The increasing incidence of initial steroid resistance in childhood nephrotic syndrome. Pediatr Nephrol. 2012;27(6):927-932.

7. Zagury A, Oliveira AL, Montalvão JA, et al. Steroid-resistant idiopathic nephrotic syndrome in children: long-term follow-up and risk factors for end-stage renal disease. J Bras Nefrol. 2013;35(3):191-199.

8. Gulati A, Bagga A, Gulati S, et al. Management of steroid resistant nephrotic syndrome. Indian Pediatr. 2019;46(1):35-47.

9. Korbet SM, Schwartz MM, Lewis EJ. Primary focal segmental glomerulosclerosis: clinical course and response to therapy. Am J Kidney Dis. 1994;23(6):773-783.

10. Burgess E. Management of focal segmental glomerulo-sclerosis: evidence-based recommendations. Kidney Int Suppl. 1999;70:S26-S32.

11. Pei Y, Cattran D, Delmore T, et al. Evidence suggesting under-treatment in adults with idiopathic focal segmental glomerulosclerosis. Am J Med. 1987;82(5):938-944.

12. Sinha A, Bagga A. Pulse steroid therapy. Indian $J$ Pediatr. 2008;75:1057-1066.

13. Mori K, Honda M, Ikeda M. Efficacy of methylprednisolone pulse therapy in steroid-resistant nephrotic syndrome. Pediatr Nephrol. 2004;19(11):1232-1236.

14. Eldin M, EL-Kersh I, Khedr M. Pulse Methylprednisolone Therapy in
Children with Resistant Nephrotic Syndrome. Alexandria Journal of Pediatrics. 1999;13(2):539-544.

15. Waldo FB, Benfield MR, Kohaut EC. Methylprednisolone treatment of patients with steroid-resistant nephrotic syndrome. Pediatr Nephrol. 1992;6(6):503-505.

16. Mendoza SA, Reznik VM, Griswold WR, et al. Treatment of steroidresistantfocal segmental glomerulosclerosis with pulsemethylprednisolone and alkylating agents. Pediatr Nephrol. 1900;4(4):303-307.

17. Lieberman KV, Tejani A. A randomized double-blind placebo-controlled trial of cyclosporine in steroid-resistant idiopathic focal segmental glomerulosclerosis in children. J Am Soc Nephrol. 1996;7(1):56-63.

18. Cattran DC, Appel GB, Hebert LA, et al. A randomized trial of cyclosporine in patients with steroid-resistant focal segmental glomerulosclerosis. Kidney Int. 1996;56(6):2220-2226.

19. Niaudet P. Treatment of childhood steroid-resistant idiopathic nephrosis with a combination of cyclosporine and prednisone. J Pediatr. 1981;125(6 Pt 1):981-986.

20. Collaborative Study Group of Sandimmun in Nephrotic Syndrome. Safety and tolerability of cyclosporin A (Sandimmun) in idiopathic nephrotic syndrome. Clin Nephrol. 1994;35(Suppl 1):S48-S60.

21. Chen LZ, Jiang XY, Lu HY, et al. [Efficacy and safety of cyclosporine A in treatment of refractory nephrotic syndrome in children: a systematic review of randomized controlled trials]. Zhonghua $\mathrm{Er} \mathrm{Ke} \mathrm{Za} \mathrm{Zhi.}$ 2019;47(12):898-903.

22. El-Husseini A, El-Basuony F, Mahmoud I, et al. Long-term effects of cyclosporine in children with idiopathic nephrotic syndrome: a singlecentre experience. Nephrol Dial Transplant. 2005;20(11):2433-2438.

23. Tenbrock K, Müller-Berghaus J, Fuchshuber A, et al. Levamisole treatment in steroid-sensitive and steroid-resistant nephrotic syndrome. Pediatr Nephrol. 2018;12(6): 459-462.

24. McCauley J, Shapiro R, Scantlebury V, et al. FK 506 in the Management of Transplant-Related Nephrotic Syndrome and Steroid-Resistant Nephrotic Syndrome. Transplant Proc. 1991;23(6):3354-3356.

25. McCauley J, Shapiro R, Ellis D. Pilot study of FK506 in the management of steroid -resistant nephrotic syndrome. Nephrol Dial Transplant. 1993;8:1286-1290.

26. Almeida MP, Almeida HA, Rosa FC. Vincristine in steroid-resistant nephrotic syndrome. Pediatr Nephrol. 1994;8(1): 79-80.

27. Goonasekera CD, Koziell AB, Hulton SA, Dillon MJ (1998) Vincristine and focal segmental sclerosis: do we need a multicentre trial? Pediatr Nephrol 12(4): 284-289.

28. Rowinsky. The Vinca Alkaloids. In: D Kufe, et al., euitor. Holland-Frei Cancer Medicine. (6th edn), BC Decker, Hamilton, Canada. 2003.

29. Vallerskog T, Gunnarsson I, Widhe M, et al. Treatment with rituximab affects both the cellular and the humoral arm of the immune system in patients with SLE. Clin Immunol. 2007;122(1):62-74.

30. Bruneau S, Dantal J. New insights into the pathophysiology of idiopathic nephrotic syndrome. Clin Immunol. 2009;133(1):13-21.

31. Peters HP, van de Kar NC, Wetzels JF. Rituximab in minimal change nephropathy and focal segmental glomerulosclerosis: report of four cases and review of the literature. Neth J Med. 2007;66(10):408-415.

32. Nakayama M, Kamei K, Nozu K, et al. Rituximab for refractory focal segmental glomerulosclerosis. Pediatr Nephrol. 2007;23(3):481-485.

33. Suri M, Tran K, Sharma AP, et al. Remission of steroid-resistant nephrotic syndrome due to focal and segmental glomerulosclerosis using rituximab. Int Urol Nephrol. 2008;40(3):807-810.

34. Prytuła A, Iijima K, Kamei K, et al. Rituximab in refractory nephrotic syndrome. Pediatr Nephrol. 2010;25(3):461-468. 
35. Gulati A, Sinha A, Jordan SC, et al. Efficacy and Safety of Treatmen with Rituximab for Difficult Steroid-Resistant and -Dependent Nephrotic Syndrome: Multicentric Report. Clin J Am Soc Nephrol. 2010;5(12):2207-2212.

36. Bagga A, Sinha A, Moudgil A. Rituximab in patients with the steroidresistant nephrotic syndrome. N Engl J Med. 2007;356(23):2751-2752.

37. Magnasco A, Ravani P, Edefonti A, et al. Rituximab in children with resistant idiopathic nephrotic syndrome. $J$ Am Soc Nephrol. 2002;23(6):1117-1124.

38. Ganji M. Using Mycophenolate Mofetil in Steroid-Resistant Nephrotic Syndrome. Iran J Kidney Dis. 2002;6:323-333.

39. Briggs WA, Choi MJ, Scheel PJ. Successful mycophenolate mofetil treatment of glomerular disease. Am J Kidney Dis. 1998;31(2):213-217.

40. Li Z, Duan C, He J, et al Mycophenolate mofetil therapy for children with steroid-resistant nephrotic syndrome. Pediatr Nephrol. 2009;25(5):883-888.

41. Latta K, von Schnakenburg C, Ehrich JH. A meta-analysis of cytotoxic treatment for frequently relapsing nephrotic syndrome in children. Pediatr Nephrol. 2001;16(3):271-282.

42. Pravitsitthikul N, Willis NS, Hodson EM, et al. Non-corticosteroid immunosuppressive medications for steroid-sensitive nephrotic syndrome in children. Cochrane Database Syst Rev. 2013;(10):CD002290.

43. Abeyagunawardena A. Treatment of steroid sensitive nephrotic syndrome. Indian J Pediatr. 2005;72(9):763-769.

44. Shah SR, Altaf A, Arshad MH, et al. Use of Cyclosporine Therapy in Steroid Resistant Nephrotic Syndrome (SRNS): A Review. Glob J Health Sci. 2015;8(4):136-141.

45. Alpay H, Yildiz N, Onar A, et al. Varicella vaccination in children with steroid-sensitive nephrotic syndrome. Pediatr Nephrol. 2002;17(3):181-183

46. Latta K, von Schnakenburg C, Ehrich JH. A meta-analysis of cytotoxic treatment for frequently relapsing nephrotic syndrome in children. Pediatr Nephrol. 2001;16(3):271-282.

47. Murphy JL, Kano HL, Chenaille PJ, et al. FatalPneumocystis pneumonia in a child treated for focal segmental glomerulosclerosis. Pediatr Nephrol. 1993;7(4):444-445.

48. Faurschou M, Sorensen IJ, Mellemkjaer L, et al. Malignancies in Wegener's granulomatosis: incidence and relation to cyclophosphamide therapy in a cohort of 293 patients. J Rheumatol. 2008;35(1):100-105.

49. Pani A, Porta C, Cosmai L, et al. Glomerular diseases and cancer: evaluation of underlying malignancy. J Nephrol. 2015;29(2):143-152.
50. Clowse ME, Copland SC, Hsieh TC, et al. Ovarian reserve diminished by oral cyclophosphamide therapy for granulomatosis with polyangiitis (Wegener's). Arthritis Care Res (Hoboken). 2011;63(12):1777-1781.

51. Clowse ME, Magder L, Petri M. Cyclophosphamide for lupus during pregnancy. Lupus. 2005;14(8):593-597.

52. Alchi B, Jayne D. Membranoproliferative glomerulonephritis. Pediatr Nephrol. 2009;25(8):1409-1418.

53. Weening JJ, D'Agati VD, Schwartz MM, et al. The classification of glomerulonephritis in systemic lupus erythematosus revisited. $J$ Am Soc Nephrol. 2004;15(2):241-250.

54. Adam FU, Torun D, Bolat F, et al. Acute renal failure due to mesangial proliferative glomerulonephritis in a pregnant woman with primary Sjögren's syndrome. Clin Rheumatol. 2006;25(1):75-79.

55. Smith RJ, Alexander J, Barlow PN, et al. New approaches to the treatment of dense deposit disease. J Am Soc Nephrol. 2007;18(9):2447-2456.

56. Sethi S, Fervenza FC, Zhang Y, et al. C3 glomerulonephritis: clinicopathological findings, complement abnormalities, glomerular proteomic profile, treatment, and follow-up. Kidney Int. 2012;82(4):465-473.

57. Bomback AS, Smith RJ, Barile GR, et al. Eculizumab for dense deposit disease and C3 glomerulonephritis. Clin J Am Soc Nephrol. 2012;7(5):748-756

58. Tarshish P, Bernstein J, Tobin JN, et al. Treatment of mesangiocapillary glomerulonephritis with alternate-day prednisone? a report of The International Study of Kidney Disease in Children. Pediatr Nephrol. 1992;6(2):123-130.

59. Cattran DC, Cardella CJ, Roscoe JM, et al. Results of a controlled drug trial in membranoproliferative glomerulonephritis. Kidney Int. $1985 ; 27(2): 436-441$.

60. Faedda R, Satta A, Tanda F, et al. Immunosuppressive Treatment of Membranoproliferative Glomerulonephritis. Nephron. 2008;67(1):59-65.

61. Bagheri N, Nemati E, Rahbar K, et al. Cyclosporine in the treatment of membranoproliferative glomerulonephritis. Archives of Iranian Medicine. 2008;11(1):26-29.

62. Haddad M, Lau K, Butani L. Remission of membranoproliferative glomerulonephritis type I with the use of tacrolimus. Pediatr Nephrol. 2007;22(10):1787-1791.

63. Jones G, Juszczak M, Kingdon E, et al. Treatment of idiopathic membranoproliferative glomerulonephritis with mycophenolate mofetil and steroids. Nephrol Dial Transplant. 2004;19(12):3160-3164. 